\title{
Decomposition Theorem for Product of Fundamental Crystals in Monomial Realization
}

\author{
Manal Alshuqayı1 and Toshiki Nakashima 2
}

\begin{abstract}
We consider a product of fundamental crystals in monomial realization. Then we shall show that the product holds crystal structure and describe how it is decomposed into irreducible crystals, which is, in general, different from the decomposition for tensor product of the fundamental crystals.
\end{abstract}

\section{Introduction}

Quantum groups $U_{q}(\mathfrak{g})$ are certain families of Hopf algebras that are deformations of universal enveloping algebras of Kac-Moody algebra $g$. The theory of crystal bases developed by M. Kashiwara [3] provides a powerful combinatorial and algebraic tool to study the representations theory of quantum groups. Roughly, we can say that crystal base is a basis for certain $U_{q}(\mathfrak{g})$-module at $q=0$. It is shown by Kashiwara that an arbitrary module in the category $\boldsymbol{O}_{\text {int }}$ [1] has unique crystal base. And a tensor product of such modules also has a crystal base, which means crystal bases hold a natural tensor product structure.

By Kashiwara operators on crystal bases, we get the graph structure on a crystal base, which is called "crystal graph". By the following simple rule of Kashiwara operators on tensor product (see sect.2):

$$
\begin{aligned}
& \tilde{e}_{i}\left(b_{1} \otimes b_{2}\right)= \begin{cases}\tilde{e}_{i} b_{1} \otimes b_{2} & \text { if } \varphi_{i}\left(b_{1}\right) \geq \varepsilon_{i}\left(b_{2}\right), \\
b_{1} \otimes \tilde{e}_{i} b_{2} & \text { if } \varphi_{i}\left(b_{1}\right)<\varepsilon_{i}\left(b_{2}\right),\end{cases} \\
& \tilde{f}_{i}\left(b_{1} \otimes b_{2}\right)= \begin{cases}\tilde{f}_{i} b_{1} \otimes b_{2} & \text { if } \varphi_{i}\left(b_{1}\right)>\varepsilon_{i}\left(b_{2}\right), \\
b_{1} \otimes \tilde{f}_{i} b_{2} & \text { if } \varphi_{i}\left(b_{1}\right) \leq \varepsilon_{i}\left(b_{2}\right),\end{cases}
\end{aligned}
$$

we know that explicit structure on tensor product of crystal bases.

Indeed, a connected component in the crystal graph of a tensor product corresponds to a simple module in the tensor product of modules. That is, let $B(\lambda)$ be a crystal of an irreducible module $V(\lambda)$ ( $\lambda$ is dominant), then, for any dominant weight $\lambda, \mu$, there exist dominant weights $\lambda_{1}, \ldots, \lambda_{k}$ such that

$$
B(\lambda) \otimes B(\mu) \cong B\left(\lambda_{1}\right) \oplus \ldots \oplus B\left(\lambda_{k}\right)
$$

and

$$
V(\lambda) \otimes V(\mu) \cong V\left(\lambda_{1}\right) \oplus \ldots \oplus V\left(\lambda_{k}\right) .
$$

\footnotetext{
${ }^{1}$ Division of Green Science and Engineering, Sophia University, Kioicho 7-1. Chiyoda-ku, Tokyo 102-8554 Japan, E-mail address: m-alshuqayr@eagle.sophia.ac.jp

2 Division of Mathematics, Sophia University, Kioicho 7-1. Chiyoda-ku, Tokyo 102-8554 Japan, E-mail address: toshiki@sophia.ac.jp
} 
There are several types of realization for crystal bases, e.g., tableaux realization, path realization, polyhedral realization, etc. Here we will treat the "monomial realization" which is introduced by Nakajima [5] and Kashiwara [3]. Let $\mathcal{M}$ be the set of Laurent monomials in the variables $Y_{i}(n)(i \in I, n \in \mathbb{Z}$, see sect.3):

$$
\mathcal{M}:=\left\{\prod_{i \in I, n \in \mathbb{Z}} Y_{i}(n)^{y_{i}(n)} ; y_{i}(n) \in \mathbb{Z} \text { vanish except finitely many }(i, n)\right\} .
$$

Then we can define the crystal structures on this set of Laurent monomials, and it is shown if a monomial $Y$ satisfies the highest condition, then the connected component in $\mathcal{M}$ including $Y$ is isomorphic to the crystal $B(w t(Y))$ corresponding to the module $V(w t(Y))$ [3] as we mentioned above. Let us denote this component by $\mathcal{M}(Y)$. Of course, $\mathcal{M}(Y) \otimes \mathcal{M}\left(Y^{\prime}\right)$ has a crystal structure. But it is not clear whether the set of products

$$
\mathcal{M}(Y) \cdot \mathcal{M}\left(Y^{\prime}\right):=\left\{M_{1} \cdot M_{2} \mid M_{1} \in \mathcal{M}(Y), M_{2} \in \mathcal{M}\left(Y^{\prime}\right)\right\},
$$

holds a crystal structure, where $M_{1} \cdot M_{2}$ means a natural product of Laurent monomials.

Let $\mathcal{M}\left(Y_{i}(n)\right)(i \in I, n \in \mathbb{Z})$ be one of monomial realizations for the fundamental crystal $B\left(\Lambda_{i}\right)$.

In this article, for type $A_{n}$ we consider the following problems:

(1) dose $\mathcal{M}\left(Y_{p}(m)\right) \cdot \mathcal{M}\left(Y_{q}(1)\right)(p, q \in I, m \in \mathbb{Z})$ hold a crystal structure?

(2) if the answer for (1) is affirmative, describe the decomposition of the crystal

$$
\mathcal{M}\left(Y_{p}(m)\right) \cdot \mathcal{M}\left(Y_{q}(1)\right) \text {. }
$$

The answer for (1) is affirmative which is shown in Propostion 5.2, and more general results are obtained in Corollary 5.3 , for any $m_{1}, m_{2}, \ldots m_{l} \in \mathbb{Z}, p_{1}, p_{2}, \ldots p_{l} \in I(l>0)$.

$$
\mathcal{M}\left(Y_{p_{1}}\left(m_{1}\right)\right) \cdot \mathcal{M}\left(Y_{p_{2}}\left(m_{2}\right)\right) \cdot \ldots \cdot \mathcal{M}\left(Y_{p_{l}}\left(m_{l}\right)\right)
$$

holds a crystal structure. Here note that there might be some monomials $M_{1}, M_{2} \in \mathcal{M}(Y)$ and $M_{1}^{\prime}, M_{2}^{\prime} \in \mathcal{M}\left(Y^{\prime}\right)$ such that $M_{1} \neq M_{1}^{\prime}, M_{2} \neq M_{2}^{\prime}$ and $M_{1} \cdot M_{2}=M_{1}^{\prime} \cdot M_{2}^{\prime}$. Therefore, it may happen:

$$
\# \mathcal{M}(Y) \cdot \mathcal{M}\left(Y^{\prime}\right)<\# \mathcal{M}(Y) \otimes \mathcal{M}\left(Y^{\prime}\right),
$$

and then one can deduce that the decomposition for $\mathcal{M}(Y) \cdot \mathcal{M}\left(Y^{\prime}\right)$ may differ from the one for $\mathcal{M}(Y) \otimes \mathcal{M}\left(Y^{\prime}\right)$. Indeed, the answer for (2) is given in Theorem 5.9 .

1. If $p+q>n$, we have

$$
\mathcal{M}\left(Y_{p}(m)\right) \cdot \mathcal{M}\left(Y_{q}(1)\right) \cong B\left(\Lambda_{p}+\Lambda_{q}\right) \oplus \bigoplus_{i=\max (p+q-n, q+1-m)}^{\min (p, q)-1} B\left(\Lambda_{p+q-i}+\Lambda_{i}\right) \oplus \mathcal{L}(m \geq n-p+2) \cdot B\left(\Lambda_{p+q-n-1}\right) .
$$

2. If $p+q \leq n$, we have

$$
\mathcal{M}\left(Y_{p}(m)\right) \cdot \mathcal{M}\left(Y_{q}(1)\right) \cong B\left(\Lambda_{p}+\Lambda_{q}\right) \oplus \bigoplus_{i=\max (1, q+1-m)}^{\min (p, q)-1} B\left(\Lambda_{p+q-i}+\Lambda_{i}\right) \oplus \mathcal{L}(m \geq q+1) \cdot B\left(\Lambda_{p+q}\right),
$$

where $\mathcal{L}(P)=1$ if $P$ is true and $\mathcal{L}(P)=0$ otherwise. For example, by this theorem, if $m$ is sufficiently large, we find that $\mathcal{M}\left(Y_{p}(m)\right) \cdot \mathcal{M}\left(Y_{q}(1)\right) \cong B\left(\Lambda_{p}\right) \otimes B\left(\Lambda_{q}\right)$. But, if not we find that 
$\mathcal{M}\left(Y_{p}(m)\right) \cdot \mathcal{M}\left(Y_{q}(1)\right)$ is not necessarily isomorphic to $B\left(\Lambda_{p}\right) \otimes B\left(\Lambda_{q}\right)$. Indeed, if $m=1$, we know that $\mathcal{M}\left(Y_{p}(m)\right) \cdot \mathcal{M}\left(Y_{q}(1)\right) \cong B\left(\Lambda_{p}+\Lambda_{q}\right) \subsetneq B\left(\Lambda_{p}\right) \otimes B\left(\Lambda_{q}\right)$.

The organization of the article is as follows:

In Section 2, we will review on quantum groups, crystal bases, crystals and there properties on tensor product. In Section 3,Nakajima's monomial realization of crystals will be introduced, which is our main subject in the article [5] [3]. In Section 4 , the explicit form of monomial realization for the fundamental crystal $B\left(\Lambda_{i}\right)$ of type $A_{n}$ will be given following [2]. In the last Section, we will state the main results, which are the answers for the questions (1) and (2). In fact, we will show that the product for fundamental crystals in monomial realization of type $A_{n}$ holds a crystal structure and describe its decomposition theorem by classifying the highest weight monomials in the product.

After uploading the article to arXiv, J.Kamnitzer notified us that in their article "Highest weight for truncated shifted Yangians and product monomial crystals," they treated the same problem for simply-laced cases. Their method for approaching the problem is some geometric way and then completely different from ours, which is purely combinatorial and direct.

Acknowledgments: We appreciate M.Nakasuji and Y.Kanakubo for there helpful comments and suggestions.

\section{Preliminaries}

Let $A=\left(a_{i j}\right)$ be the Cartan matrix for a simple Lie algebra $\mathfrak{g}$ and let $\mathbb{Q}(q)$ be the rational function field in $q$.

Definition. 2.1. Let $\mathfrak{g}$ be a finite-dimensional simple Lie algebra with a Cartan sub-algebra $\mathrm{t}$, the set of simple roots $\left\{\alpha_{i} \in t^{*}\right\}_{i \in I}$ and the set of simple coroots $\left\{h_{i} \in t\right\}_{i \in I}$, where $I=\{1, \ldots, n\}$. We take an inner product $($,$) on t^{*}$ such that $\left(\alpha_{i}, \alpha_{i}\right) \in \mathbb{Z}_{>0}$ and $\left\langle h_{i}, \lambda\right\rangle=2\left(\alpha_{i}, \lambda\right) /\left(\alpha_{i}, \alpha_{i}\right)$ for $\lambda_{i} \in t^{*}$. Let $\left\{\Lambda_{i}\right\}_{i \in I}$ be the dual base of $\left\{h_{i}\right\}$ and set $P:=\sum \mathbb{Z} \Lambda_{i}$ and $P^{\vee}:=\sum \mathbb{Z} h_{i}$. Then the $q$-analogue $U_{q}(\mathfrak{g})$ is the algebra over $\mathbb{Q}(q)$ generated by $e_{i}, f_{i}, k_{i}^{ \pm 1}(i \in I=\{1, \ldots, n\})$ satisfying the relations

$$
\begin{gathered}
k_{i} k_{i}^{-1}=1=k_{i}^{-1} k_{i}, k_{i} k_{j}=k_{j} k_{i}, \\
k_{i} e_{j} k_{i}^{-1}=q_{i}^{a_{i j}} e_{j}, \\
k_{i} f_{j} k_{i}^{-1}=q_{i}^{a_{i j}} f_{j}, \\
e_{i} f_{j}-f_{j} e_{i}=\delta_{i j} \frac{k_{i}-k_{j}^{-1}}{q_{i}-q_{i}^{-1}}, \\
\sum_{s=0}^{1-a_{i j}}(-1)^{s}\left[\begin{array}{c}
1-a_{i j} \\
s
\end{array}\right]_{i} e_{i}^{1-a_{i j}-s} e_{j} e_{i}^{s}=0,(i \neq j), \\
\sum_{s=0}^{1-a_{i j}}(-1)^{s}\left[\begin{array}{c}
1-a_{i j} \\
s
\end{array}\right]_{i} f_{i}^{1-a_{i j}-s} f_{j} f_{i}^{s}=0,(i \neq j),
\end{gathered}
$$

where $\delta_{i j}$ is the Kronecker delta. 
Notation. 2.2. Set now for all $\left\{\alpha_{i} \mid i=1,2, \ldots, n\right\}$

$$
q_{i}=q^{\left(\alpha_{i}, \alpha_{i}\right)}
$$

and (for all $a \in \mathbb{Z}$ )

$$
[a]_{i}:=\frac{q_{i}^{a}-q_{i}^{-a}}{q_{i}-q_{i}^{-1}} \text {. }
$$

Define $[n]_{i} !:=[n]_{i}[n-1]_{i} \ldots[2]_{i}[1]_{i}$, and $\left[\begin{array}{l}a \\ n\end{array}\right]_{i}:=\frac{[a]_{i} !}{[n]_{i} ![a-n]_{i} !}\left(a, n \in \mathbb{Z}_{\geq 0}, a \geq n\right)$.

Let $M$ be a finite dimensional $U_{q}(\mathfrak{g})$-module and for $\lambda \in P$ set $M_{\lambda}=\left\{v \in M \mid q^{h} v=q^{\langle h, \lambda\rangle}(\forall h \in\right.$ $\left.P^{\vee}\right)$, which is called a weight space of weight $\lambda$. Then we know that $M=\bigoplus_{\lambda \in P} M_{\lambda}$.

Definition. 2.3. Let $M=\bigoplus_{\lambda \in P} M_{\lambda}$ be a finite dimensional $U_{q}(\mathfrak{g})$-module. For each $i \in I$, every weight vector $u \in M_{\lambda}(\lambda \in w t(M))$ is written in the form

$$
u=u_{0}+f_{i} u_{1}+\ldots+f_{i}^{(N)} u_{N},
$$

where $N \in \mathbb{Z}_{\geq 0}$ and $u_{k} \in M_{\lambda+k \alpha_{i}} \cap \operatorname{Ker} e_{i}$ for any $k=0,1, \ldots, N$. Here, each $u_{k}$ in the expression is uniquely determined by $u$, and $u_{k} \neq 0$ only if $\lambda\left(h_{i}\right)+k>0$. The Kashiwara operators $\tilde{e}_{i}, \tilde{f}_{i} \in \operatorname{End}_{\mathbb{Q}(q)}(M)(i \in I)$ are defined for $u=\sum_{k=0}^{N} f_{i}^{(k)} u_{k} \in M_{\lambda}$ by

$$
\tilde{e}_{i} u=\sum_{k=1}^{N} f_{i}^{(k-1)} u_{k}, \quad \tilde{f}_{i} u=\sum_{k=0}^{N} f_{i}^{(k+1)} u_{k} .
$$

Let $A$ be the subring of $\mathbb{Q}(q)$ defined by $A=\{f(q) / g(q) \mid f(q), g(q) \in \mathbb{Q}[q], g(q) \neq 0\}$.

Definition. 2.4. Let $M$ be a finite dimensional $U_{q}(\mathfrak{g})$-module and $L$ be a free $A$-submodule. $L$ is called a crystal lattice if

1. L generates $M$ as a vector space over $\mathbb{Q}(q)$,

2. $L=\bigoplus_{\lambda \in P} L_{\lambda}$, where $L_{\lambda}=L \cap M_{\lambda}$ for all $\lambda \in P$,

3. $\tilde{e}_{i} L \subset L, \tilde{f}_{i} L \subset L$ for all $i \in I$.

Definition. 2.5. A crystal base of a finite dimensional $U_{q}(\mathfrak{g})$-module $M$ is a pair $(L, B)$ such that

1. L is a crystal lattice of $M$,

2. B is an $\mathbb{Q}$-basis of $L / q L$,

3. $B=\bigsqcup_{\lambda \in P} B_{\lambda}$, where $B_{\lambda}=B \cap\left(L_{\lambda} / q L_{\lambda}\right)$,

4. $\tilde{e}_{i} B \subset B \cup\{0\}, \tilde{f_{i}} B \subset B \cup\{0\}$ for all $i \in I$,

5. for any $b, b^{\prime} \in B$ and $i \in I$, we have $\tilde{f}_{i} b=b^{\prime}$ if and only if $b=\tilde{e}_{i} b^{\prime}$.

Let $P_{+}:=\left\{\sum_{i=1}^{n} m_{i} \Lambda_{i} \mid m_{i} \in \mathbb{Z}_{\geq 0}(\forall i \in I)\right\}$ be the set of dominant weights. For any finite dimensional irreducible $U_{q}(\mathfrak{g})$-module $L$, there exists a unique dominant weight $\lambda \in P_{+}$such that $L \cong V(\lambda)$, where $V(\lambda):=U_{q}(\mathfrak{g}) / \sum_{i \in I} U_{q}(\mathfrak{g}) e_{i}+\sum_{i \in I} U_{q}(\mathfrak{g})\left(t_{i}-q_{i}^{\left\langle h_{i}, \lambda\right\rangle}\right)+\sum_{i \in I} U_{q}(\mathfrak{g}) f_{i}^{1+\left\langle h_{i}, \lambda\right\rangle}$. 
Here let $\pi_{\lambda}:=U_{q}(\mathfrak{g}) \rightarrow V(\lambda)$ be the natural projection and set $u_{\lambda}:=\pi_{\lambda}(1)$, which is the highest weight vector of $V(\lambda)$.

Let us define for $\lambda \in P_{+}$,

$$
\begin{aligned}
L(\lambda) & :=\sum_{\substack{i_{1}, \ldots, i_{l} \in I \\
l \geq 0}} A \tilde{f}_{i_{1}} \ldots \tilde{f}_{i_{l}} u_{\lambda}, \\
B(\lambda) & :=\left\{\tilde{f}_{i_{1}} \ldots \tilde{f}_{i_{l}} u_{\lambda} \bmod q L(\lambda) \mid i_{1}, \ldots, i_{l} \in I, l \geq 0\right\} \backslash\{0\} .
\end{aligned}
$$

Theorem. 2.6. [8, 9] The pair $(L(\lambda), B(\lambda))$ is a crystal base of $V(\lambda)$.

Theorem. 2.7. [8, 9] Let $M_{j}$ be a finite dimensional $U_{q}(\mathfrak{g})$-module and let $\left(L_{j}, B_{j}\right)$ be a crystal basis of $M_{j}(j=1,2)$. Set $L=L_{1} \otimes_{A_{0}} L_{2}$ and $B=B_{1} \times B_{2}$.

Then $(L, B)$ is a crystal basis of $M_{1} \otimes_{A_{0}} M_{2}$, where the action of Kashiwara operators $\tilde{e}_{i}$ and $\tilde{f}_{i}$ on $B(i \in I)$ is given by

$$
\begin{aligned}
& \tilde{e}_{i}\left(b_{1} \otimes b_{2}\right)=\left\{\begin{array}{lll}
\tilde{e}_{i} b_{1} \otimes b_{2} & \text { if } & \varphi_{i}\left(b_{1}\right) \geq \varepsilon_{i}\left(b_{2}\right), \\
b_{1} \otimes \tilde{e}_{i} b_{2} & \text { if } & \varphi_{i}\left(b_{1}\right)<\varepsilon_{i}\left(b_{2}\right),
\end{array}\right. \\
& \tilde{f}_{i}\left(b_{1} \otimes b_{2}\right)=\left\{\begin{array}{lll}
\tilde{f}_{i} b_{1} \otimes b_{2} & \text { if } & \varphi_{i}\left(b_{1}\right)>\varepsilon_{i}\left(b_{2}\right), \\
b_{1} \otimes \tilde{f}_{i} b_{2} & \text { if } & \varphi_{i}\left(b_{1}\right) \leq \varepsilon_{i}\left(b_{2}\right),
\end{array}\right.
\end{aligned}
$$

and we have

$$
\begin{aligned}
w t\left(b_{1} \otimes b_{2}\right) & =w t\left(b_{1}\right)+w t\left(b_{2}\right), \\
\varepsilon_{i}\left(b_{1} \otimes b_{2}\right) & =\max \left(\varepsilon_{i}\left(b_{1}\right), \varepsilon_{i}\left(b_{2}\right)-\left\langle h_{i}, w t\left(b_{1}\right)\right\rangle\right), \\
\varphi_{i}\left(b_{1} \otimes b_{2}\right) & =\max \left(\varphi_{i}\left(b_{1}\right), \varphi_{i}\left(b_{2}\right)-\left\langle h_{i}, w t\left(b_{2}\right)\right\rangle\right) .
\end{aligned}
$$

Here, we write $b_{1} \otimes b_{2}$ for $\left(b_{1}, b_{2}\right) \in B_{1} \times B_{2}$ and we understand $b_{1} \otimes 0=0 \otimes b_{2}=0$.

Definition. 2.8. [7] Let $I$ be a finite index set and let $A=\left(a_{i j}\right)_{i, j \in I}$ be a Cartan matrix and $P$ a corresponding weight lattice. Crystal associated with the Cartan matrix $A$ is a set $B$ together with the maps wt $: B \rightarrow P, \tilde{e}_{i}, \tilde{f}_{i}: B \rightarrow B \cup\{0\}$, and $\varepsilon_{i}, \varphi_{i}: B \rightarrow \mathbb{Z} \cup\{-\infty\}(i \in I)$ satisfying the following properties:

1. $\varphi_{i}(b)=\varepsilon_{i}(b)+\left\langle h_{i}, w t(b)\right\rangle$ for all $i \in I$,

2. $w t\left(\tilde{e}_{i} b\right)=w t b+\alpha_{i}$ if $\tilde{e}_{i} b \in B$,

3. $w t\left(\tilde{f_{i}} b\right)=w t b-\alpha_{i}$ if $\tilde{f}_{i} b \in B$,

4. $\varepsilon_{i}\left(\tilde{e}_{i} b\right)=\varepsilon_{i}(b)-1, \varphi_{i}\left(\tilde{e}_{i} b\right)=\varphi_{i}(b)+1$ if $\tilde{e}_{i} b \in B$,

5. $\varepsilon_{i}\left(\tilde{f_{i}} b\right)=\varepsilon_{i}(b)+1, \varphi_{i}\left(\tilde{f_{i}} b\right)=\varphi_{i}(b)-1$ if $\tilde{f}_{i} b \in B$,

6. $\tilde{f}_{i} b=b^{\prime}$ if and only if $b=\tilde{e}_{i} b^{\prime}$ for $b, b^{\prime} \in B, i \in I$,

7. if $\varphi_{i}(b)=-\infty$ for $b \in B$, then $\tilde{e}_{i} b=\tilde{f}_{i} b=0$.

Definition. 2.9. Take $B$ as the set of vertices and the $I$-colored arrows on $B$ by

$$
b \stackrel{i}{\longrightarrow} b^{\prime} \text { if and only if } \tilde{f}_{i} b=b^{\prime}(i \in I) .
$$

Then $B$ is given an $I$-colored oriented graph structure called the crystal graphs of $M$. 


\section{Nakajima's monomials}

In this section, we recall the crystal structure on the set of monomials discovered by $\mathrm{H}$. Nakajima [5]. Our exposition follows that of M.Kashiwara [3].

Let $\mathcal{M}$ be the set of Laurent monomials in the variables $Y_{i}(n)(i \in I, n \in \mathbb{Z})$ :

$$
\mathcal{M}:=\left\{\prod_{i \in I, n \in \mathbb{Z}} Y_{i}(n)^{y_{i}(n)} ; y_{i}(n) \in \mathbb{Z} \text { vanish except finitely many }(i, n)\right\} .
$$

We shall define a crystal structure of crystal on $\mathcal{M}$. Let $c=\left(c_{i j}\right)_{i \neq j \in I}$, be a set of integers such that

$$
c_{i j}+c_{j i}=1
$$

We set

$$
A_{i}(n)=Y_{i}(n) Y_{i}(n+1) \prod_{j \neq i} Y_{j}\left(n+c_{j i}\right)^{\left\langle h_{j}, \alpha_{i}\right\rangle} .
$$

For a monomial $M=\prod_{i \in I, n \in \mathbb{Z}} Y_{i}(n)^{y_{i}(n)} \in \mathcal{M}$, we set

$$
\begin{aligned}
w t(M) & =\sum_{i}\left(\sum_{n} Y_{i}(n)\right) \Lambda_{i}, \\
\varphi_{i}(M) & =\max \left\{\sum_{k \leq n} y_{i}(k) ; n \in \mathbb{Z}\right\}, \\
\varepsilon_{i}(M) & =\max \left\{-\sum_{k>n} y_{i}(k) ; n \in \mathbb{Z}\right\} .
\end{aligned}
$$

We define

$$
\begin{aligned}
& \tilde{f}_{i}(M)=\left\{\begin{array}{ccc}
0 & \text { If } & \varphi_{i}(M)=0, \\
A_{i}\left(n_{f}\right)^{-1} M & \text { If } & \varphi_{i}(M)>0,
\end{array}\right. \\
& \tilde{e}_{i}(M)=\left\{\begin{array}{ccc}
0 & \text { If } & \varepsilon_{i}(M)=0, \\
A_{i}\left(n_{e}\right) M & \text { If } & \varepsilon_{i}(M)>0,
\end{array}\right.
\end{aligned}
$$

where

$$
\begin{aligned}
n_{f} & =\min \left\{n ; \varphi_{i}(M)=\sum_{k \leq n} y_{i}(k)\right\}, \\
& =\min \left\{n ; \varepsilon_{i}(M)=-\sum_{k>n} y_{i}(k)\right\}, \\
n_{e} & =\max \left\{\mathrm{n} ; \varphi_{i}(M)=\sum_{k \leq n} y_{i}(k)\right\}, \\
& =\max \left\{n ; \varepsilon_{i}(M)=-\sum_{k>n} y_{i}(k)\right\} .
\end{aligned}
$$

Note that $y_{i}\left(n_{f}\right)>0, y_{i}\left(n_{f}+1\right) \leq 0$ and $y_{i}\left(n_{e}+1\right)<0, y_{i}\left(n_{e}\right) \geq 0$.

Let us denote by $\mathcal{M}_{c}$ the crystal $\mathcal{M}$ associated with c.

We shall call $I$-crystal when we want to emphasize the index set $I$ of simple roots.

For an $I$-crystal $B$ and a subset $J$ of $I$, let us denote by $\Psi_{J}(B)$ the $J$-crystal $B$ with the induced crystal structure from $B$.

A crystal $B$ is called semi-normal if for each $i \in I$ the $\{i\}$-crystal $\Psi_{\{i\}}(B)$ is a crystal associated with a finite dimensional module. This is equivalent to saying that $\varepsilon_{i}(b)=\max \{n \in$ $\left.N ; \tilde{e}_{i}^{n} b \neq 0\right\}$ and $\varphi_{i}(b)=\max \left\{n \in N ; \tilde{f}_{i}^{n} b \neq 0\right\}$ for any $b \in B$ and $i \in I$.

$A$ crystal $B$ is called normal if for any subset $J$ of $I$ such that $\left\{\alpha_{i} ; i \in J\right\}$ is a set of simple roots for finite-dimensional semisimple Lie algebra $\mathfrak{g}_{\mathrm{J}}, \Psi_{J}(B)$ is the crystal associated 
with a finite dimensional $U_{q}\left(\mathfrak{g}_{\mathrm{J}}\right)$-module, where $U_{q}\left(\mathfrak{g}_{\mathrm{J}}\right)$ is the associated quantum group with $\left\{\alpha_{i} ; i \in J\right\}$.

Proposition. 3.1. $\mathcal{M}_{c}$ is a semi-normal crystal.

For a family of integers $\left(m_{i}\right)_{i \in I}$, let us set $c^{\prime}=\left(c^{\prime}{ }_{i j}\right)_{i \neq j \in I}$, by $c^{\prime}{ }_{i j}=c_{i j}+m_{i}-m_{j}$. Then the map $Y_{i}(n) \longmapsto Y_{i}\left(n+m_{i}\right)$ gives a crystal isomorphism $\mathcal{M}_{c} \longrightarrow \mathcal{M}_{c^{\prime}}$. Hence if the Dynkin diagram has no loop, the isomorphism class of the crystal $\mathcal{M}_{c}$ does not depend on the choice of $c$.

Theorem. 3.2. [3] For a highest weight vector $M \in \mathcal{M}_{c}$, the connected component of $\mathcal{M}_{c}$ containing $M$ is isomorphic to $B(w t(M))$.

Corollary. 3.3. [3] $\mathcal{M}_{c}$ is a normal crystal.

\section{Proposition. 3.4.}

1. For each $i \in I, \mathcal{M}$ is isomorphic to a crystal graph of a $U_{q}\left(\mathfrak{s I}_{2}\right)$-module.

2. Let $M$ be a monomial with weight $\lambda$, such that $\tilde{e}_{i} M=0$ for all $i \in I$, and let $\mathcal{M}(\lambda)$ be the connected component of $\mathcal{M}$ containing $M$. Then there exists a crystal isomorphism

$$
\mathcal{M}(\lambda) \stackrel{\sim}{\longrightarrow} B(\lambda) \quad \text { given by } \quad M \longmapsto v_{\lambda} .
$$

Remark. Not that, the condition $\tilde{e}_{i} M=0$ for all $i \in I$, is called the highest weight condition, which is, equivalent to the condition $\varphi_{i}(M)=0$ for all $i \in I$.

\section{Monomial Realization of the Fundamental Crystals of Type $A_{n}$}

We refer to [2]. Let $I=\{1, \ldots, n\}$ and let $A=\left(a_{i j}\right)_{i, j \in I}$ be the Cartan matrix of type $A_{n}$. Here, the entries of $A$ are given by

$$
a_{i j}=\left\{\begin{array}{rll}
2 & \text { if } & i=j, \\
-1 & \text { if } & |i-j|=1, \\
0 & & \text { otherwise. }
\end{array}\right.
$$

Let $U_{q}(\mathfrak{g})=U_{q}\left(s l_{n+1}\right)$ the corresponding quantum group. For simplicity, we take the set $C=\left(c_{i j}\right)_{i \neq j}$ to be

$$
c_{i j}= \begin{cases}0 & \text { if } i>j, \\ 1 & \text { if } i<j,\end{cases}
$$

and set $Y_{0}(m)^{ \pm 1}=Y_{n+1}(m)^{ \pm 1}=1$ for all $m \in \mathbb{Z}$. Then for $i \in I$ and $m \in \mathbb{Z}$, we have

$$
A_{i}(m)=Y_{i}(m) Y_{i}(m+1) Y_{i-1}(m+1)^{-1} Y_{i+1}(m)^{-1} .
$$

To characterize $\mathcal{M}(\lambda)$, we first focus on the case when $\lambda=\Lambda_{k}$. Let $M_{0}=Y_{k}(m)$ for $m \in \mathbb{Z}$. Hence $\tilde{e}_{i} M_{0}=0$ for all $i \in I$ and the connected component containing $M_{0}$ is isomorphic to $B\left(\Lambda_{k}\right)$ over $U_{q}(\mathfrak{g})$. For simplicity, we will take $M_{0}=Y_{k}(1)$, even if that does not make much difference. 
Proposition. 4.1. For $k=1, \ldots, n$, let $M_{0}=Y_{k}(1)$ be the monomial of weight $\Lambda_{k}$ such that $\tilde{e}_{i} M_{0}=0$ for all $i \in I$. Then the connected component $\mathcal{M}\left(M_{0}\right)=\mathcal{M}\left(Y_{k}(1)\right)$ of $M$ containing $M_{0}$ is characterized as

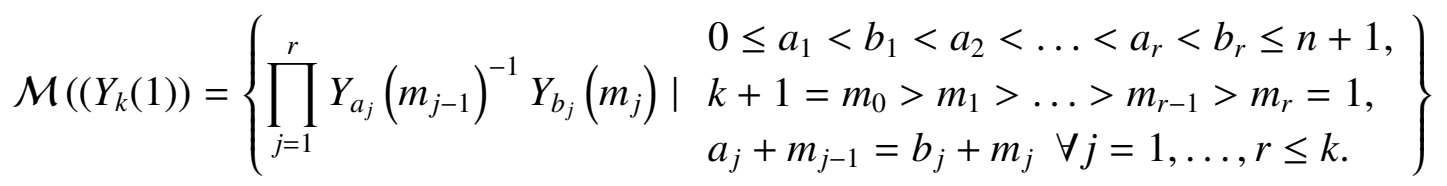

Remark. If we take $M_{0}=Y_{k}(N)$, then we have only to modify the condition for $m_{j}$ 's as follows:

$$
k+N=m_{0}>m_{1}>\ldots>m_{r-1}>m_{r}=N .
$$

For $i \in I$ and $m \in \mathbb{Z}$, we introduce new variables

$$
X_{i}(m)=Y_{i-1}(m+1)^{-1} Y_{i}(m) .
$$

Note that $X_{n+1}(m)=\frac{1}{Y_{n}(m+1)}$. Using this notation, every monomial

$$
M=\prod_{j=1}^{r} Y_{a_{j}}\left(m_{j-1}\right)^{-1} Y_{b_{j}}\left(m_{j}\right) \in M\left(Y_{k}(1)\right),
$$

may be written as

$$
M=\prod_{j=1}^{r} X_{a_{j}+1}\left(m_{j-1}-1\right) X_{a_{j}+2}\left(m_{j-1}-2\right) \ldots X_{b_{j}}\left(m_{j}\right) .
$$

For example, we have $Y_{k}(N)=X_{1}(k+N-1) X_{2}(k+N-2) \ldots X_{k}(N)$. Now, it is straightforward to verify that we have another characterization of the crystal $\mathcal{M}\left(Y_{k}(1)\right)$.

Corollary. 4.2. For $k=1, \ldots n$ we have

$$
\mathcal{M}\left(Y_{k}(N)\right)=\left\{X_{i_{1}}(k+N-1) X_{i_{2}}(k+N-2) \ldots X_{i_{k}}(N) \mid 1 \leq i_{1}<i_{2}<\ldots<i_{k} \leq n+1\right\} .
$$

Example. 4.3. Let $\mathfrak{g}=A_{5}$, and $c_{i j}=\left\{\begin{array}{lll}0 & \text { if } & i>j \\ 1 & \text { if } & i<j\end{array}\right.$.

1. The crystal $B\left(\Lambda_{5}\right) \cong \mathcal{M}\left(Y_{5}(m)\right)$ is given as follows.

$$
\begin{array}{r}
\mathcal{M}\left(Y_{5}(m)\right)=\left\{X_{i_{1}}(4+m) X_{i_{2}}(3+m) X_{i_{3}}(2+m) X_{i_{4}}(1+m) X_{i_{5}}(m) \mid 1 \leq i_{1}<i_{2}<i_{3}<i_{4}<i_{5} \leq 6\right\}, \\
Y_{5}(m) \stackrel{\tilde{f}_{5}}{\longrightarrow} Y_{5}(m+1)^{-1} Y_{4}(m+1) \stackrel{\tilde{f}_{4}}{\longrightarrow} Y_{4}(m+2)^{-1} Y_{3}(m+2) \stackrel{\tilde{f}_{3}}{\longrightarrow} Y_{3}(m+3)^{-1} Y_{2}(m+3) \\
Y_{1}(m+5)^{-1} \stackrel{\tilde{f}_{1}}{\longleftarrow} Y_{2}(m+4)^{-1} Y_{1}(m+4)
\end{array}
$$

Figure 3 
2. The crystal $B\left(\Lambda_{2}\right) \cong \mathcal{M}\left(Y_{2}(1)\right)$ is given as follows.

$$
\mathcal{M}\left(Y_{2}(1)\right)=\left\{X_{i_{1}}(2) X_{i_{2}}(1) \mid 1 \leq i_{1}<i_{2} \leq 6\right\},
$$

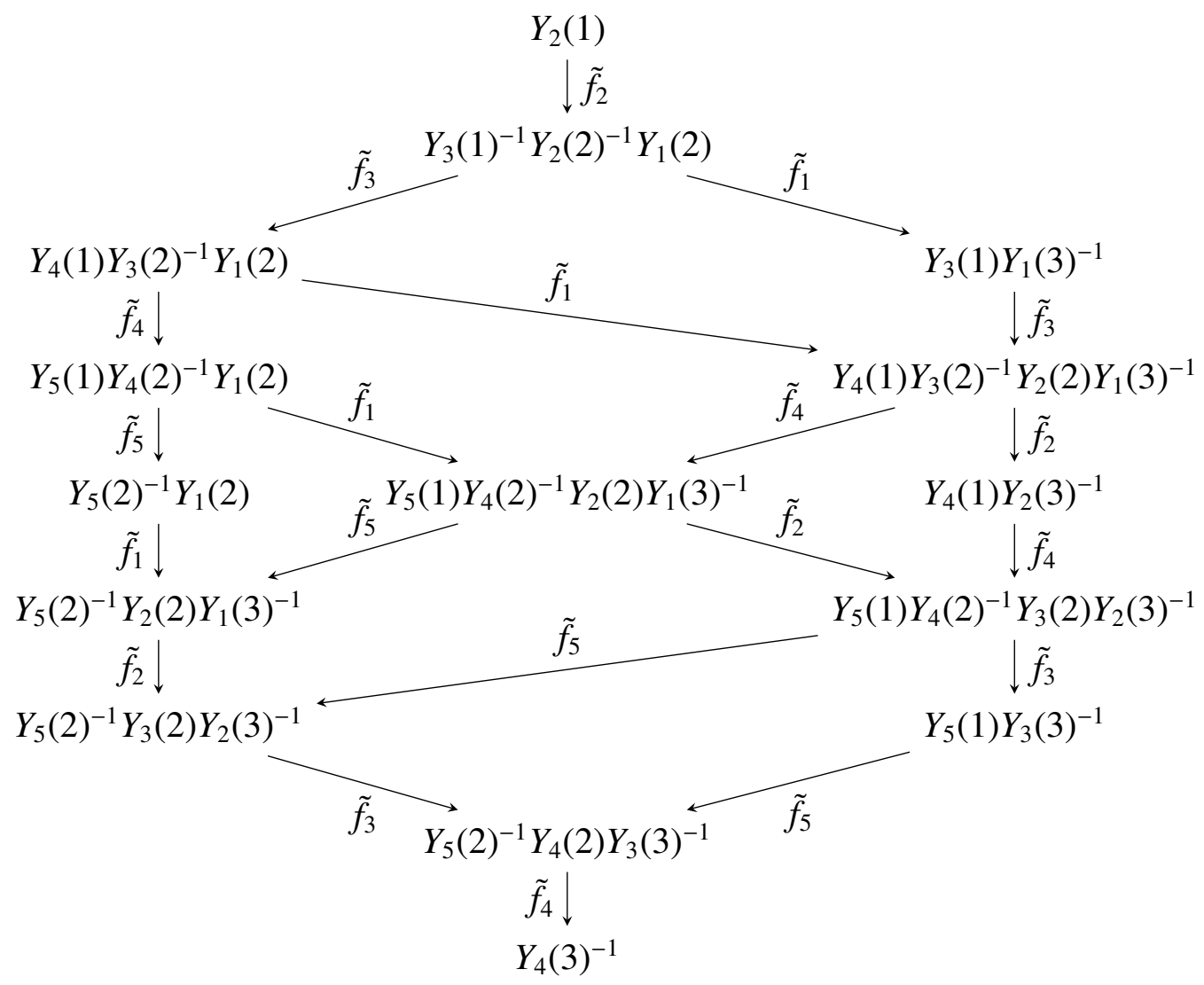

Figure 4

\section{Product of Fundamental Crystals in Monomial Realization}

In this section we study the crystal structures of product for fundamental crystals in monomial realization of type $A_{n}$. We will show that such a product has a crystal structure and we introduce the decomposition theorem for product of fundamental crystals in monomial realization.

\subsection{The Product $\mathcal{M}\left(Y_{p}(m)\right) \cdot \mathcal{M}\left(Y_{q}(1)\right)$}

We use the same notation as the previous sections.

Lemma. 5.1. Let $M_{1} \in \mathcal{M}\left(Y_{p}(m)\right), M_{2} \in \mathcal{M}\left(Y_{q}(1)\right)$ with $m \geq 1$, and $p, q \in\{1, \ldots, n\}$. Then for any $i \in I$, we have

$$
\begin{aligned}
& \tilde{e}_{i}\left(M_{1} \cdot M_{2}\right) \in \mathcal{M}\left(Y_{p}(m)\right) \cdot \mathcal{M}\left(Y_{q}(1)\right) \cup\{0\}, \\
& \tilde{f}_{i}\left(M_{1} \cdot M_{2}\right) \in \mathcal{M}\left(Y_{p}(m)\right) \cdot \mathcal{M}\left(Y_{q}(1)\right) \cup\{0\} .
\end{aligned}
$$


Proof. By the explicit description in Corollary 4.2 , we can write

$$
\begin{aligned}
& M_{1}=X_{i_{1}}(p+m-1) X_{i_{2}}(p+m-2) \ldots X_{i_{p}}(m), \\
& M_{2}=X_{j_{1}}(q) X_{j_{2}}(q-1) \ldots X_{j_{q}}(1) .
\end{aligned}
$$

The factor $Y_{i}(l)^{-1}(l \in \mathbb{Z})$ can appear at most once in $M_{1}$ and $M_{2}$ by Proposition 4.1 respectively then we obtain

$$
\tilde{e}_{i}\left(M_{1} \cdot M_{2}\right)=\left\{\begin{array}{l}
\left(\tilde{e}_{i} M_{1}\right) \cdot M_{2}, \\
M_{1} \cdot\left(\tilde{e}_{i} M_{2}\right), \\
0 .
\end{array}\right.
$$

Indeed, since if both $Y_{i}\left(l_{1}\right)^{-1}$ appears in $M_{1}$ and $Y_{i}\left(l_{2}\right)^{-1}$ appears in $M_{2}$, by the definition of $\tilde{e}_{i}$ we have

$$
\begin{aligned}
\tilde{e}_{i}\left(M_{1} \cdot M_{2}\right) & =A_{i}\left(l_{1}\right)\left(M_{1} \cdot M_{2}\right) \text { or } A_{i}\left(l_{2}\right)\left(M_{1} \cdot M_{2}\right) \\
& =\left(\tilde{e}_{i} M_{1}\right) \cdot M_{2} \text { or } M_{1} \cdot\left(\tilde{e}_{i} M_{2}\right) .
\end{aligned}
$$

If one of them appears in $M_{1}$ or $M_{2}$, we also have

$$
\tilde{e}_{i}\left(M_{1} \cdot M_{2}\right)=\left(\tilde{e}_{i} M_{1}\right) \cdot M_{2} \text { or } M_{1} \cdot\left(\tilde{e}_{i} M_{2}\right) .
$$

Similarly, by Proposition 4.1 we have

$$
\tilde{f}_{i}\left(M_{1} \cdot M_{2}\right)=\left\{\begin{array}{l}
\left(\tilde{f}_{i} M_{1}\right) \cdot M_{2}, \\
M_{1} \cdot\left(\tilde{f_{i}} M_{2}\right), \\
0 .
\end{array}\right.
$$

Proposition. 5.2. The product $\mathcal{M}\left(Y_{p}(m)\right) \cdot \mathcal{M}\left(Y_{q}(1)\right)$ possesses the crystal structure and it is decomposed into a direct sum of crystals, that is, there exist dominant integral weights $\lambda_{1}, \lambda_{2}, \ldots, \lambda_{k} \in P_{+}$such that

$$
\mathcal{M}\left(Y_{p}(m)\right) \cdot \mathcal{M}\left(Y_{q}(1)\right) \cong B\left(\lambda_{1}\right) \oplus B\left(\lambda_{2}\right) \oplus \ldots \oplus B\left(\lambda_{k}\right) .
$$

Proof. The former half of the statement is clear from Lemma 5.1, And then we know that $\mathcal{M}\left(Y_{p}(m)\right) \cdot \mathcal{M}\left(Y_{q}(1)\right)$ is a union of some connected components. Each connected component is isomorphic to some $B(\lambda)\left(\lambda \in P_{+}\right)$since the crystal $\mathcal{M}_{c}$ is normal by Corollary 3.3 .

Corollary. 5.3. For any $m_{1}, m_{2}, \ldots m_{l} \in \mathbb{Z}, p_{1}, p_{2}, \ldots p_{l} \in I(l>0)$.

$$
\mathcal{M}\left(Y_{p_{1}}\left(m_{1}\right)\right) \cdot \mathcal{M}\left(Y_{p_{2}}\left(m_{2}\right)\right) \cdot \ldots \cdot \mathcal{M}\left(Y_{p_{l}}\left(m_{l}\right)\right)
$$

holds a crystal structure.

Lemma. 5.4. We have the following decomposition for the tensor product of fundamental crystals $B\left(\Lambda_{p}\right)$ and $B\left(\Lambda_{q}\right)$ :

1. For $p+q>n$,

$$
\begin{gathered}
B\left(\Lambda_{p}\right) \otimes B\left(\Lambda_{q}\right) \cong B\left(\Lambda_{\max (p, q)}+\Lambda_{\min (p, q)}\right) \oplus B\left(\Lambda_{\max (p, q)+1}+\Lambda_{\min (p, q)-1}\right) \oplus \ldots \\
\oplus B\left(\Lambda_{n}+\Lambda_{p+q-n}\right) \oplus B\left(\Lambda_{p+q-n-1}\right) .
\end{gathered}
$$


2. For $p+q \leq n$,

$$
\begin{gathered}
B\left(\Lambda_{p}\right) \otimes B\left(\Lambda_{q}\right) \cong B\left(\Lambda_{\max (p, q)}+\Lambda_{\min (p, q)}\right) \oplus B\left(\Lambda_{\max (p, q)+1}+\Lambda_{\min (p, q)-1}\right) \oplus \ldots \\
\oplus B\left(\Lambda_{p+q-1}+\Lambda_{1}\right) \oplus B\left(\Lambda_{p+q}\right)
\end{gathered}
$$

Proof. cf. [4].

Lemma. 5.5. Any connected component in $\mathcal{M}\left(Y_{p}(m)\right) \cdot \mathcal{M}\left(Y_{q}(1)\right)$ appears in $\mathcal{M}\left(Y_{p}(m)\right) \otimes$ $\mathcal{M}\left(Y_{q}(1)\right)$.

Proof. We may show that for each dominant weight $\lambda$ in $w t\left(\left(\mathcal{M}\left(Y_{p}(m)\right) \otimes \mathcal{M}\left(Y_{q}(1)\right)\right)\right.$ we find $B(\lambda) \subset \mathcal{M}\left(Y_{p}(m)\right) \otimes \mathcal{M}\left(Y_{q}(1)\right)$, since

$$
w t\left(\left(\mathcal{M}\left(Y_{p}(m)\right) \cdot \mathcal{M}\left(Y_{q}(1)\right)\right)=w t\left(\mathcal{M}\left(Y_{p}(m)\right) \otimes \mathcal{M}\left(Y_{q}(1)\right)\right) .\right.
$$

To find all the dominant weights in $\mathcal{M}\left(Y_{p}(m)\right) \otimes \mathcal{M}\left(Y_{q}(1)\right)$, we may consider $B\left(\Lambda_{p}\right) \otimes B\left(\Lambda_{q}\right)$ as in [4]. Let $\left(i_{1}, i_{2}, \ldots, i_{p}\right)$ be an element in $B\left(\Lambda_{p}\right)$ like as a column tableau with the entries $i_{1}, i_{2}, \ldots, i_{p}$ satisfying $1 \leq i_{1}<i_{2}<i_{3} \ldots<i_{p} \leq n+1$. Take $u=\left(i_{1}, \ldots, i_{p}\right) \in B\left(\Lambda_{p}\right)$ and $v=\left(j_{1}, \ldots, j_{q}\right) \in B\left(\Lambda_{q}\right)$ whose weights are $w t(u)=\epsilon_{i_{1}}+\ldots+\epsilon_{i_{p}}, w t(v)=\epsilon_{j_{1}}+\ldots+\epsilon_{j_{q}}$, where $\epsilon_{1}=\Lambda_{1}, \epsilon_{i}=\Lambda_{i}-\Lambda_{i-1}(i=1,2, \ldots, n)$ and $\epsilon_{n+1}=-\epsilon_{1}-\epsilon_{2} \ldots-\epsilon_{n}$. Note that $\left\{\epsilon_{1} \ldots \epsilon_{n}\right\}$ are linearly independent. Now we assume $p \geq q$ without loss of generality. Note that

$$
\Lambda_{k}=\epsilon_{1}+\ldots+\epsilon_{k},(1 \leq k \leq n) .
$$

If $w t(u)+w t(v)$ is dominant, it should includes $\epsilon_{1}$ once or twice and then we have the following cases:

1. If it includes $\epsilon_{1}$ once then $w t(u)+w t(v)=\Lambda_{k}$ for some $k$.

2. If it includes $\epsilon_{1}$ twice then $w t(u)+w t(v)=\Lambda_{k}+\Lambda_{l}$ for some $k$ and $l(k \leq l)$.

Consider the case 1

First, we assume $p+q>n$, then

$$
\Lambda_{k}=w t(u)+w t(v)=\epsilon_{i_{1}}+\ldots+\epsilon_{i_{p}}+\epsilon_{j_{1}}+\ldots+\epsilon_{j_{q}} .
$$

If neither $i_{p}$ nor $j_{q}$ is $n+1$, some coefficients of $\epsilon_{l}(1 \leq l \leq n)$ are 2 , which means

$$
w t(u)+w t(v) \neq \Lambda_{k}
$$

If both $i_{p}$ and $j_{q}$ are $n+1$, it is trivial that $w t(u)+w t(v)$ is never dominant.

If $i_{p} \neq n+1$ and $j_{q}=n+1$, then $w t(u)$ and $w t(v)$ is

$$
\begin{aligned}
& w t(u)=\epsilon_{i_{1}}+\ldots+\epsilon_{i_{p}}, \\
& w t(v)=\epsilon_{j_{1}}+\ldots+\epsilon_{j_{q-1}}-\left(\epsilon_{1}+\ldots+\epsilon_{n}\right) .
\end{aligned}
$$

If $w t(u)+w t(v)=\Lambda_{k}=\epsilon_{1}+\ldots+\epsilon_{k}$, then we have

$$
\epsilon_{1}+\ldots+\epsilon_{k}=\epsilon_{i_{1}}+\ldots+\epsilon_{i_{p}}+\epsilon_{j_{1}}+\ldots+\epsilon_{j_{q-1}}-\left(\epsilon_{1}+\ldots+\epsilon_{n}\right) .
$$

Then we obtain $k+n=p+q-1$ and then $k=p+q-n-1$. So, we get $q>k$. Indeed, if $q \leq k=$ $p+q-n-1$, then $0 \leq p-n-1$, which can not happen. In this case $u=\left(i_{1}, \ldots, i_{p}\right)=(1, \ldots, p)$ 
and $v=\left(j_{1}, \ldots, j_{q-1}, j_{q}\right)=(1, \ldots, k, p+1, \ldots, n, n+1)$ gives $w t(u)+w t(v)=\Lambda_{k}=\Lambda_{p+q-n-1}$. By Lemma 5.4, the component $B\left(\Lambda_{p+q-n-1}\right)$ appears in $B\left(\Lambda_{p}\right) \otimes B\left(\Lambda_{q}\right)$.

The case $i_{p}=n+1$ and $j_{q} \neq n+1$ is done similarly. Indeed, $\left(i_{1}, \ldots, i_{p-1}\right)=(1, \ldots, p-1)$ and $\left(j_{1}, \ldots, j_{q}\right)=(1, \ldots, k, p, \ldots, n)$ gives $w t(u)+w t(v)=\Lambda_{k}=\Lambda_{p+q-n-1}$.

Second, we assume $p+q \leq n$. If one of $i_{p}$ or $j_{q}$ is $n+1, w t(u)+w t(v)$ is not dominant. Thus, we know that neither $i_{p}$ nor $j_{q}$ is $n+1$. The assumption $w t(u)+w t(v)=\Lambda_{k}$ means $p+q=k$. Indeed, if $u=\left(i_{1}, \ldots, i_{P}\right)=(1, \ldots, p)$ and $v=\left(j_{1}, \ldots, j_{q}\right)=(p+1, \ldots, p+q)$, then we have $w t(u)+w t(v)=\Lambda_{p+q}$. By Lemma 5.4 the component $B\left(\Lambda_{p+q}\right)$ appears in $B\left(\Lambda_{p}\right) \otimes B\left(\Lambda_{q}\right)$.

Now consider the case 2. In this case, $\epsilon_{1}$ appears twice in $w t(u)+w t(v)$, which means neither $i_{P}$ nor $j_{q}$ is $n+1$. Then we know that $p+q=k+l$.

First, assume $p+q>n$. Now, we claim $k \leq q \leq p \leq l$. Let us assume $k>q$. Then we have

$$
\Lambda_{k}+\Lambda_{l}=2 \varepsilon_{1}+\ldots+2 \epsilon_{k}+\epsilon_{k+1}+\ldots+\epsilon_{l},
$$

and

$$
w t(u)+w t(v)=\epsilon_{i_{1}}+\ldots+\epsilon_{i_{p}}+\epsilon_{j_{1}}+\ldots+\epsilon_{j_{q}},
$$

which shows the number of coefficient 2 in (5.3) is at most $q$ and then it contradicts with (5.2). Thus, we have $k \leq q$ and $k \leq q \leq p \leq l$ since $k+l=p+q$.

Such $(k, l)$ are: $(q, p),(q-1, p+1),(q-2, p+2), \ldots,(p+q-n, n)$. All of them appear in the decomposition as in Lemma 5.4. Indeed, in this case $k=q-i, l=p+i(0 \leq i \leq$ $n-p), u=\left(i_{1}, \ldots, i_{p}\right)=(1, \ldots, p)$ and $v=\left(j_{1}, \ldots, j_{q}\right)=(1, \ldots, q-i, p+1, \ldots, p+i)$ gives $w t(u)+w t(v)=\Lambda_{p+i}+\Lambda_{q-i}(0 \leq i \leq n-p)$.

Next, we assume $p+q \leq n$. By arguing similarly to the previous case we find the possibility of $(k, l)$ are: $(q, p),(q-1, p+1), \ldots,(1, p+q)$. All of them appears in the decomposition as in Lemma 5.4. Indeed, in the case $k=q-i, l=p+i(0 \leq i \leq q-1), u=\left(i_{1}, \ldots, i_{p}\right)=(1, \ldots, p)$ and $v=\left(j_{1}, \ldots, j_{q}\right)=(1, \ldots, q-i, p+1, \ldots, p+i)$ gives $w t(u)+w t(v)=\Lambda_{p+i}+\Lambda_{q-i}(0 \leq i \leq q-1)$.

Lemma. 5.6. Let $M_{1} \in \mathcal{M}\left(Y_{p}(m)\right), M_{2} \in \mathcal{M}\left(Y_{q}(1)\right)$ with $m \geq 1$ and $p, q \in\{1, \ldots, n\}$. If $M_{1} \cdot M_{2}$ is the highest weight vector then $M_{1}=Y_{p}(m)$.

Proof. The general form of $M_{1}$ is given in Corollary 4.2 ,

$$
M_{1}=X_{i_{1}}(p+m-1) X_{i_{2}}(p+m-2) \ldots X_{i_{p}}(m)\left(1 \leq i_{1}<\ldots<i_{P} \leq n+1\right) .
$$

Assume that there is $j \in\{0,1, \ldots, p-1\}$ such that $i_{j}+1<i_{j+1}$ (we set $i_{0}=0$ ) and take $j_{0}$ is the biggest one among such $j^{\prime}$ s, which means there are gaps in the sequence $\left(i_{1} \ldots i_{p}\right)$ and $j_{0}$ is the last gap. Note that $j_{0}<p$. Set $i_{j_{0}+1}=h$, then there is

$$
X_{h}\left(p+m-j_{0}-1\right)=\frac{Y_{h}\left(p+m-j_{0}-1\right)}{Y_{h-1}\left(p+m-j_{0}\right)},
$$

in $M_{1}$ and by the assumption that $M_{1} \cdot M_{2}$ is the highest, we should have the factor $Y_{h-1}(\alpha)$ in $M_{2}$, and by the explicit form of $M_{2}$, we can find the factors

$$
\frac{Y_{h-1}(\alpha)}{Y_{h-2}(\alpha+1)} \cdot \frac{Y_{h^{\prime}}(\alpha-1)}{Y_{h^{\prime}-1}(\alpha)}\left(h^{\prime}>h\right)
$$

in $M_{2}$ and we obtain

$$
\alpha \geq p+m-j_{0} .
$$


We have $\frac{1}{Y_{h^{\prime}-1}(\alpha)}$ in $M_{2}$, which means there is the factor $Y_{h^{\prime}-1}(\beta)$ in $M_{1}$ by the assumption that $M_{1} \cdot M_{2}$ is the highest. Since $h=i_{j_{0}+1}$ is the gap in $M_{1}$ and after $h$ the indices $i_{j}$ 's are consecutive, $h^{\prime}-1$ should be $i_{p}$.

Thus, we have $Y_{h^{\prime}-1}(\beta)=Y_{i_{p}}(m)$, and

$$
m \geq \alpha
$$

then from (5.4) and (5.5), we get

$$
m \geq \alpha \geq p+m-j_{0} \geq m+1,
$$

which is a contradiction. Then, we obtain $i_{j}=j$ (for all $\left.j=1, \ldots, p\right)$ and then $M_{1}=X_{1}(p+m-$ 1)... $X_{p}=Y_{p}(m)$.

\subsection{Decomposition of Monomial Product}

Lemma. 5.7. Let $M$ be a monomial in $\mathcal{M}\left(Y_{q}(1)\right)$ and $p \in\{1, \ldots, n\}$.

1. If $w t(M)=\Lambda_{q}$, then $M=X_{1}(q) \ldots X_{q}(1)=Y_{q}(1)$.

2. If $\max (1, p+q-n) \leq i<\min (p, q)$ and $w t(M)=\Lambda_{p+q-i}+\Lambda_{i}-\Lambda_{p}$, then

$$
\begin{aligned}
M & =X_{1}(q) \ldots X_{i}(q-i+1) \cdot X_{p+1}(q-i) \ldots X_{p+q-i}(1) \\
& =Y_{i}(q-i+1) \cdot \frac{Y_{p+q-i}(1)}{Y_{p}(q-i+1)} .
\end{aligned}
$$

3. If $p+q>n$ and $w t(M)=\Lambda_{p+q-n-1}-\Lambda_{p}$, then

$$
\begin{aligned}
M & =X_{1}(q) \ldots X_{p+q-n-1}(n-p+2) \cdot X_{p+1}(n-p+1) \ldots X_{n+1}(1) \\
& =\frac{Y_{p+q-n-1}(n-p+2)}{Y_{p}(n-p+2)} .
\end{aligned}
$$

4. If $p+q \leq n$ and $w t(M)=\Lambda_{p+q}-\Lambda_{p}$, then $M=X_{p+1}(q) \ldots X_{p+q}(1)=\frac{Y_{p+q}(1)}{Y_{p}(q+1)}$

Proof. It is known that all weight multiplicities of $B\left(\Lambda_{q}\right)$ is free. And it is clear that the monomial (5.6), (5.7), (5.8) and (5.9) are in $\mathcal{M}\left(Y_{q}(1)\right)$ by Corollary 4.2, and have the corresponding weights.

Theorem. 5.8. For $M_{1} \in \mathcal{M}\left(Y_{p}(m)\right), M_{2} \in \mathcal{M}\left(Y_{q}(1)\right)$ with $m \geq 1$ and $p, q \in\{1, \ldots, n\}$, assume that $M_{1} \cdot M_{2}$ is the highest weight vector if and only if the following cases occur:

1. $w t(M)=\Lambda_{p}+\Lambda_{q}$, we have $M_{1}=Y_{p}(m), M_{2}=Y_{q}(1)$.

2. $w t\left(M_{1} \cdot M_{2}\right)=\Lambda_{p+q-i}+\Lambda_{i}$ with $\max (1, p+q-n) \leq i \leq \min (p, q)-1$, and $m \geq q-i+1$, we have $M_{1}=Y_{p}(m)$,

$$
M_{2}=X_{1}(q) \ldots X_{i}(q-i+1) X_{p+1}(q-i) \ldots X_{p+q-i}(1)=Y_{i}(q-i+1) \cdot \frac{Y_{p+q-i}(1)}{Y_{p}(q-i+1)} .
$$

3. $w t\left(M_{1} \cdot M_{2}\right)=\Lambda_{p+q-n-1}$ with $p+q>n$, and $m \geq n-p+2$, we have $M_{1}=Y_{p}(m)$,

$$
M_{2}=X_{1}(q) \ldots X_{p+q-n-1}(n-p+2) X_{p+1}(n-p+1) \ldots X_{n+1}(1)=\frac{Y_{p+q-n-1}(n-p+2)}{Y_{p}(n-p+2)} \text {. }
$$

4. $w t\left(M_{1} \cdot M_{2}\right)=\Lambda_{p+q}$ with $p+q \leq n$, and $m \geq q+1$, we have $M_{1}=Y_{p}(m)$,

$$
M_{2}=X_{p+1}(q) \ldots \ldots X_{p+q}(1)=\frac{Y_{p+1}(1)}{Y_{p}(q+1)} \text {. }
$$


Proof. First, we assume that $M_{1} \cdot M_{2}$ is the highest weight vector then by Lemma 5.6, $M_{1}=$ $Y_{p}(m)$. We know by Lemma 5.5 that any connected component $B(\lambda)$ in $\mathcal{M}\left(Y_{p}(m)\right) \cdot \mathcal{M}\left(Y_{q}(1)\right)$ appears in $\mathcal{M}\left(Y_{p}(m)\right) \otimes \mathcal{M}\left(Y_{q}(1)\right)$. Suppose $p+q>n$. Thus, by Lemma 5.4 we can write

$$
\begin{aligned}
B(\lambda) \subset B\left(\Lambda_{\max (p, q)}\right. & \left.+\Lambda_{\min (p, q)}\right) \oplus B\left(\Lambda_{\max (p, q)+1}+\Lambda_{\min (p, q)-1}\right) \oplus \ldots \\
& \oplus B\left(\Lambda_{n}+\Lambda_{p+q-n}\right) \oplus B\left(\Lambda_{p+q-n-1}\right) .
\end{aligned}
$$

1. We know that if $M_{1}=Y_{p}(m)$ and $M_{2}=Y_{q}(1)$, then $M_{1} \cdot M_{2}$ is always a highest weight vector in $\mathcal{M}\left(Y_{p}(m)\right) \cdot \mathcal{M}\left(Y_{q}(1)\right)$ for any $m \geq 1$.

2. In the previous case, we have already consider the case $\Lambda_{\max (p, q)}+\Lambda_{\min (p, q)}$. Then we may see the highest weight $\Lambda_{p+q-i}+\Lambda_{i}(\max (1, p+q-n) \leq i \leq \min (p, q)-1)$. Then $w t\left(M_{2}\right)=\Lambda_{p+q-i}+\Lambda_{i}-\Lambda_{p}$. By Lemma 5.7, the unique possibility of $M_{2}$ is

$$
M_{2}=Y_{i}(q-i+1) \cdot \frac{Y_{p+q-i}(1)}{Y_{p}(q-i+1)},
$$

then

$$
M_{1} \cdot M_{2}=Y_{p}(m) \cdot Y_{i}(q-i+1) \cdot \frac{Y_{p+q-i}(1)}{Y_{p}(q-i+1)},
$$

by the highest condition, we get $m \geq q-i+1$.

3. If $w t\left(M_{1} \cdot M_{2}\right)=\Lambda_{p+q-n-1}$ with $p+q>n$, then the unique possibility of $M_{2}$ is

$$
M_{2}=\frac{Y_{p+q-n-1}(n-p+2)}{Y_{p}(n-p+2)},
$$

then

$$
M_{1} \cdot M_{2}=Y_{p}(m) \cdot \frac{Y_{p+q-n-1}(n-p+2)}{Y_{p}(n-p+2)},
$$

by the highest condition, we get $m \geq n-p+2$.

4. We assume $p+q \leq n$, then by Lemma5.5 the component $B(\lambda)$ in $\mathcal{M}\left(Y_{p}(m)\right) \cdot \mathcal{M}\left(Y_{q}(1)\right)$ appears in $\mathcal{M}\left(Y_{p}(m)\right) \otimes \mathcal{M}\left(Y_{q}(1)\right)$. Thus, by Lemma 5.4 we can write

$$
\begin{aligned}
B(\lambda) \subset B\left(\Lambda_{\max (p, q)}+\right. & \left.\Lambda_{\min (p, q)}\right) \oplus B\left(\Lambda_{\max (p, q)+1}+\Lambda_{\min (p, q)-1}\right) \oplus \ldots \\
& \oplus B\left(\Lambda_{p+q-1}+\Lambda_{1}\right) \oplus B\left(\Lambda_{p+q}\right) .
\end{aligned}
$$

If $w t\left(M_{1} \cdot M_{2}\right)=\Lambda_{p+q}$, this case it done by similarly as the previous case.

On the contrary, if we assume that we have the cases it is trivial that $M_{1} \cdot M_{2}$ is the highest.

For a statement $P$, let $\mathcal{L}(P)= \begin{cases}1 & \text { if } P \text { is true }, \\ 0 & \text { if } P \text { is false. }\end{cases}$

\section{Theorem. 5.9.}

1. If $p+q>n$, we have

$$
\mathcal{M}\left(Y_{p}(m)\right) \cdot \mathcal{M}\left(Y_{q}(1)\right) \cong B\left(\Lambda_{p}+\Lambda_{q}\right) \oplus \bigoplus_{i=\max (p+q-n, q+1-m)}^{\min (p, q)-1} B\left(\Lambda_{p+q-i}+\Lambda_{i}\right) \oplus \mathcal{L}(m \geq n-p+2) \cdot B\left(\Lambda_{p+q-n-1}\right) .
$$


2. If $p+q \leq n$, we have

$$
\mathcal{M}\left(Y_{p}(m)\right) \cdot \mathcal{M}\left(Y_{q}(1)\right) \cong B\left(\Lambda_{p}+\Lambda_{q}\right) \oplus \bigoplus_{i=\max (1, q+1-m)}^{\min (p, q)-1} B\left(\Lambda_{p+q-i}+\Lambda_{i}\right) \oplus \mathcal{L}(m \geq q+1) \cdot B\left(\Lambda_{p+q}\right) .
$$

Proof. By Theorem 5.8, we know that $B\left(\Lambda_{p}+\Lambda_{q}\right)$ always appears in $\mathcal{M}\left(Y_{p}(m)\right) \cdot \mathcal{M}\left(Y_{q}(1)\right)$ for any $m \geq 1$. For $\max (1, p+q-n) \leq i \leq \min (p, q)-1, B\left(\Lambda_{p+q-i}+\Lambda i\right)$ appears in $\mathcal{M}\left(Y_{p}(m)\right)$. $\mathcal{M}\left(Y_{q}(1)\right)$ if and only if $i \geq q-m+1$.

Then, we have the condition

$$
\max (1, p+q-n, q+1-m) \leq i \leq \min (p, q)-1 \text {. }
$$

If $p+q>n$, the component $B\left(\Lambda_{p+q-n-1}\right)$ appears in $\mathcal{M}\left(Y_{p}(m)\right) \cdot \mathcal{M}\left(Y_{q}(1)\right)$ if and only if $m \geq n-p+2$. If $p+q \leq n$, the component $B\left(\Lambda_{p+q}\right)$ appears in $\mathcal{M}\left(Y_{p}(m)\right) \cdot \mathcal{M}\left(Y_{q}(1)\right)$ if and only if $m \geq q+1$.

Example. 5.10. From Example 4.3, we know the crystal graphs of $\mathcal{M}\left(Y_{2}(1)\right)$ and $\mathcal{M}\left(Y_{5}(m)\right)$ in $A_{5}$.

1. Let $p=5, q=2$, then $Y_{5}(m) \cdot Y_{2}(1) \in \mathcal{M}\left(Y_{5}(m)\right) \cdot \mathcal{M}\left(Y_{2}(1)\right), p+q=7>n=5$,

(a) if $m=1$, we have

$$
\begin{aligned}
\mathcal{M}\left(Y_{5}(1)\right) \cdot \mathcal{M}\left(Y_{2}(1)\right) & \cong B\left(\Lambda_{5}+\Lambda_{2}\right) \oplus \bigoplus_{i=\max (2,2)}^{\min (5,2)-1} B\left(\Lambda_{p+q-i}+\Lambda_{i}\right) \oplus \mathcal{L}(1 \geq 2) \cdot B\left(\Lambda_{p+q-n-1}\right) \\
& \cong B\left(\Lambda_{5}+\Lambda_{2}\right) \oplus \bigoplus_{i=2}^{1} B\left(\Lambda_{5+2-i}+\Lambda_{i}\right), \\
\mathcal{M}\left(Y_{5}(1)\right) \cdot \mathcal{M}\left(Y_{2}(1)\right) & \cong B\left(\Lambda_{5}+\Lambda_{2}\right),
\end{aligned}
$$

(b) if $m=2$, we have

$$
\begin{aligned}
\mathcal{M}\left(Y_{5}(2)\right) \cdot \mathcal{M}\left(Y_{2}(1)\right) & \cong B\left(\Lambda_{5}+\Lambda_{2}\right) \oplus \bigoplus_{i=\max (2,1)}^{\min (5,2)-1} B\left(\Lambda_{p+q-i}+\Lambda_{i}\right) \oplus \mathcal{L}(2 \geq 2) \cdot B\left(\Lambda_{p+q-n-1}\right) \\
& \cong B\left(\Lambda_{5}+\Lambda_{2}\right) \oplus \bigoplus_{i=2}^{1} B\left(\Lambda_{5+2-i}+\Lambda_{i}\right) \oplus B\left(\Lambda_{1}\right) \\
\mathcal{M}\left(Y_{5}(2)\right) \cdot \mathcal{M}\left(Y_{2}(1)\right) & \cong B\left(\Lambda_{5}+\Lambda_{2}\right) \oplus B\left(\Lambda_{1}\right) .
\end{aligned}
$$

2. Let $p=q=2$, then $Y_{2}(m) \cdot Y_{2}(1) \in \mathcal{M}\left(Y_{2}(m)\right) \cdot \mathcal{M}\left(Y_{2}(1)\right) \mathrm{m} p+q=4 \leq n=5$,

(a) if $m=1$, we have

$$
\begin{aligned}
\mathcal{M}\left(Y_{2}(1)\right) \cdot \mathcal{M}\left(Y_{2}(1)\right) & \cong B\left(\Lambda_{2}+\Lambda_{2}\right) \oplus \bigoplus_{i=\max (1,2)}^{\min (2,2)-1} B\left(\Lambda_{p+q-i}+\Lambda_{i}\right) \oplus \mathcal{L}(1 \geq 3) \cdot B\left(\Lambda_{p+q}\right), \\
\mathcal{M}\left(Y_{2}(1)\right) \cdot \mathcal{M}\left(Y_{2}(1)\right) & \cong B\left(2 \Lambda_{2}\right) \oplus \bigoplus_{i=2}^{1} B\left(\Lambda_{2+2-i}+\Lambda_{i}\right) \\
& \cong B\left(2 \Lambda_{2}\right),
\end{aligned}
$$


(b) if $m=3$, we have

$$
\begin{aligned}
\mathcal{M}\left(Y_{2}(3)\right) \cdot \mathcal{M}\left(Y_{2}(1)\right) & \cong B\left(\Lambda_{2}+\Lambda_{2}\right) \oplus \bigoplus_{i=\max (1,0)}^{\min (2,2)-1} B\left(\Lambda_{p+q-i}+\Lambda_{i}\right) \oplus \mathcal{L}(3 \geq 3) \cdot B\left(\Lambda_{p+q}\right), \\
\mathcal{M}\left(Y_{2}(3)\right) \cdot \mathcal{M}\left(Y_{2}(1)\right) & \cong B\left(2 \Lambda_{2}\right) \oplus \bigoplus_{i=1}^{1} B\left(\Lambda_{2+2-i}+\Lambda_{i}\right) \oplus B\left(\Lambda_{4}\right) \\
& \cong B\left(2 \Lambda_{2}\right) \oplus B\left(\Lambda_{3}+\Lambda_{1}\right) \oplus B\left(\Lambda_{4}\right) .
\end{aligned}
$$

3. Let $p=2, q=5$, then $Y_{5}(m) \cdot Y_{2}(1) \in \mathcal{M}\left(Y_{5}(m)\right) \cdot \mathcal{M}\left(Y_{2}(1)\right), p+q=7>n=5$,

(a) if $m=1$, we have

$$
\begin{aligned}
\mathcal{M}\left(Y_{2}(1)\right) \cdot \mathcal{M}\left(Y_{5}(1)\right) & \cong B\left(\Lambda_{2}+\Lambda_{5}\right) \oplus \bigoplus_{i=\max (2,5)}^{\min (5,2)-1} B\left(\Lambda_{p+q-i}+\Lambda_{i}\right) \oplus \mathcal{L}(1 \geq 5) \cdot B\left(\Lambda_{p+q-n-1}\right) \\
& \cong B\left(\Lambda_{2}+\Lambda_{5}\right) \oplus \bigoplus_{i=5}^{1} B\left(\Lambda_{2+5-i}+\Lambda_{i}\right), \\
\mathcal{M}\left(Y_{2}(1)\right) \cdot \mathcal{M}\left(Y_{5}(1)\right) & \cong B\left(\Lambda_{2}+\Lambda_{5}\right),
\end{aligned}
$$

(b) if $m=5$, we have

$$
\begin{aligned}
\mathcal{M}\left(Y_{2}(5)\right) \cdot \mathcal{M}\left(Y_{5}(1)\right) & \cong B\left(\Lambda_{2}+\Lambda_{5}\right) \oplus \bigoplus_{i=\max (2,1)}^{\min (5,2)-1} B\left(\Lambda_{p+q-i}+\Lambda_{i}\right) \oplus \mathcal{L}(5 \geq 5) \cdot B\left(\Lambda_{p+q-n-1}\right) \\
& \cong B\left(\Lambda_{2}+\Lambda_{5}\right) \oplus \bigoplus_{i=2}^{1} B\left(\Lambda_{2+5-i}+\Lambda_{i}\right) \oplus B\left(\Lambda_{1}\right) \\
\mathcal{M}\left(Y_{2}(5)\right) \cdot \mathcal{M}\left(Y_{5}(1)\right) & \cong B\left(\Lambda_{2}+\Lambda_{5}\right) \oplus B\left(\Lambda_{1}\right) .
\end{aligned}
$$




\section{References}

[1] J.Hong. S-J.Kang, "Introduction to Quantum Groups and Crystal Bases," American Mathematical Society (2002).

[2] S-J.Kang, J-A.Kim, D-U.Shin, "Monomial realization of crystal basas for special linear algebras," Journal of Algebra 247(2004)629-624.

[3] M.Kashiwara, "Realization of Crystals," in: Contemp. Math., vol. 325,Amer. Math. Soc., 2003, pp. 133-139.

[4] T.Nakashima, "Crystal base a generalization of the Littlewood-Richardson rule for the classsical Lie algebras,” Commun. Math. Phys. 154 (1993), no. 2, 215-243.

[5] H. Nakajima, " $t$-Analogs of $q$-Characters of Quantum affine Algebras of Type $A_{N}, D_{N}$," in: Contemp. Math., vol.325, Amer. Math. Soc., 2003,pp. 141-160.

[6] M.Kashiwara, T.Nakashima, "Crystal Graphs for Representations of the $q$-Analogue of Classical Lie Algebras,” J. of Algebra, 165(1994), 295-345.

[7] M.Kashiwara, “Crystal base and Littelmann's refined Demazure character formula," Duke Math. J. 71(1993)-839-858.

[8] M.Kashiwara, "Crystalizing the $q$-analogue of universal enveloping algebras," Comm. Math. Phys. 133(1990), 249-260.

[9] M.Kashiwara, "On crystal bases of the $q$-analogue of universal enveloping algebras," Duke Math. J. 63(1991), 456-516. 